\title{
Sex-specific relationships between adverse childhood experiences and chronic obstructive pulmonary disease in five states
}

This article was published in the following Dove Press journal:

International Journal of COPD

26 September 2014

Number of times this article has been viewed

\author{
Timothy J Cunningham' \\ Earl S Ford' \\ Janet B Croft ${ }^{\prime}$ \\ Melissa T Merrick ${ }^{2}$ \\ Italia $\vee$ Rolle $^{3}$ \\ Wayne H Giles'
}

'Division of Population Health, National Center for Chronic Disease Prevention and Health Promotion, Centers for Disease Control and Prevention, Atlanta, GA, USA; ${ }^{2}$ Division of Violence Prevention, National Center for Injury Prevention and Control, Centers for Disease Control and Prevention, Atlanta, GA, USA ${ }^{3}$ Office on Smoking and Health, National Center for Chronic Disease Prevention and Health Promotion, Centers for Disease Control and Prevention, Atlanta, GA, USA
Correspondence: Timothy J

Cunningham

Centers for Disease Control and Prevention, 4770 Buford Highway, Mailstop F78, Atlanta, GA 3034I, USA

Tel +l 7704885397

Fax +I 7704885965

Email tjcunningham@cdc.gov
Purpose: Adverse childhood experiences (ACEs) before age 18 have been repeatedly associated with several chronic diseases in adulthood such as depression, heart disease, cancer, diabetes, and stroke. We examined sex-specific relationships between individual ACEs and the number of ACEs with chronic obstructive pulmonary disease (COPD) in the general population.

Materials and methods: Data from 26,546 women and 19,015 men aged $\geq 18$ years in five states of the 2011 Behavioral Risk Factor Surveillance System were analyzed. We used loglinear regression to estimate prevalence ratios (PRs) and their corresponding 95\% confidence intervals (CIs) for the relationship of eight ACEs with COPD after adjustment for age group, race/ethnicity, marital status, educational attainment, employment, asthma history, health insurance coverage, and smoking status.

Results: Some $63.8 \%$ of women and $62.2 \%$ of men reported $\geq 1$ ACE. COPD was reported by $4.9 \%$ of women and $4.0 \%$ of men. In women, but not in men, there was a higher likelihood of COPD associated with verbal abuse ( $\mathrm{PR}=1.30,95 \% \mathrm{CI}: 1.05,1.61)$, sexual abuse ( $\mathrm{PR}=1.69$, 95\% CI: 1.36, 2.10), living with a substance abusing household member ( $\mathrm{PR}=1.49,95 \% \mathrm{CI}$ : $1.23,1.81$ ), witnessing domestic violence ( $\mathrm{PR}=1.40,95 \% \mathrm{CI}: 1.14,1.72$ ), and parental separation/divorce ( $\mathrm{PR}=1.47,95 \% \mathrm{CI}: 1.21,1.80$ ) during childhood compared to those with no individual ACEs. Reporting $\geq 5$ ACEs (PR $=2.08,95 \%$ CI: $1.55,2.80$ ) compared to none was associated with a higher likelihood of COPD among women only.

Conclusion: ACEs are related to COPD, especially among women. These findings underscore the need for further research that examines sex-specific differences and the possible mechanisms linking ACEs and COPD. This work adds to a growing body of research suggesting that ACEs may contribute to health problems later in life and suggesting a need for program and policy solutions.

Keywords: chronic obstructive pulmonary disease, childhood, abuse, sex

\section{Introduction}

Chronic obstructive pulmonary disease (COPD), a group of progressive, debilitating respiratory conditions that includes emphysema, chronic bronchitis, and small airway disease, which cause airflow blockage and breathing-related problems, is a major public health problem. ${ }^{1}$ In 2010 , there were 10.3 million physician office visits, 1.5 million emergency department visits, and 699,000 hospital discharges for COPD. ${ }^{1}$ Although tobacco cigarette smoking contributes to about $80 \%$ of COPD deaths, ${ }^{2}$ considerable proportions of COPD occur among nonsmokers. ${ }^{3}$ The childhood social environment has been suggested as a determinant of COPD for over 50 years and may partially explain the occurrence of COPD among nonsmokers., ${ }^{4,5}$ 
A growing body of epidemiologic evidence indicates that exposure to household dysfunction and childhood maltreatment including physical, sexual, and emotional abuse and neglect is common ${ }^{6-8}$ and that exposure to these experiences during childhood may result in toxic stress that places people at greater risk for poor health across the life course. ${ }^{6}$ It is hypothesized that adverse childhood experiences (ACEs) may lead to chronic diseases through emotional and psychophysiological responses that include dysregulation of the hypothalamic-pituitary-adrenal axis and through behavioral responses such as smoking. ${ }^{9-13}$ Previous studies have shown that self-reported ACEs are associated with several chronic diseases such as COPD,${ }^{14}$ depression, ${ }^{15}$ heart disease, ${ }^{16}$ cancer, ${ }^{17,18}$ diabetes, ${ }^{7}$ and stroke. ${ }^{7}$

Considering that the previous findings on ACEs and COPD came from one study of health maintenance organization members who were mostly middle class, white adults in California, ${ }^{14}$ confirming these findings in the general population is critical to better understanding the etiology of COPD and to develop prevention and intervention strategies for those at risk. It also remains unknown whether the relationship between ACEs and COPD is influenced by sex. Previous studies have found a higher prevalence of COPD among women compared to men and suggest differential susceptibility to COPD by sex. ${ }^{19-22}$ Furthermore, sex differences in the relationship between ACEs and smoking status in adulthood were identified in one previous study. ${ }^{13}$ Therefore, we examined sex-specific relationships between individual ACEs and the number of ACEs with COPD in the general population of five states in this study. We also assessed whether smoking status explained the relationship of ACEs to COPD.

\section{Methods}

\section{Study design and population}

The Behavioral Risk Factor Surveillance System (BRFSS) is a state-based, random-digit-dialed telephone survey of non-institutionalized, US adults aged 18 years or older, which is administered annually by state health departments with assistance from the US Centers for Disease Control and Prevention (CDC).$^{23}$ The 2011 BRFSS data used in this analysis reflect changes in weighting methodology (raking) and include both landline and cell phone respondents. ${ }^{23}$ BRFSS includes questions on sociodemographic characteristics, chronic diseases, health behaviors, and access to health care. Five states (Minnesota, Montana, Vermont, Washington, and Wisconsin) implemented the 2011 ACE module and COPD question.
Cooperation rates and response rates for BRFSS are calculated using standards set by the American Association of Public Opinion Research (http://www.aapor.org/ standard definitions $2 . h t m)$. The cooperation rate, which is defined as the number of completed interviews divided by the number of eligible respondents who were successfully reached by an interviewer, for each of the five states was 85.9\% (Minnesota), 77.2\% (Montana), 75.5\% (Vermont), $66.9 \%$ (Washington), and $72.7 \%$ (Wisconsin). The response rate, which is defined as the number of respondents who completed the survey as a proportion of all eligible and likely eligible persons, for each of the five states was $51.9 \%$ (Minnesota), 52.8\% (Montana), 49.9\% (Vermont), 44.3\% (Washington), and $55.1 \%$ (Wisconsin). Of 30,732 women and 22,104 men participating in the five states, we analyzed data from 26,546 women and 19,015 men with complete records for the variables included in main analyses. This study was exempt from human subjects review as the data were obtained from public-use surveillance datasets.

\section{ACEs}

The ACEs BRFSS module to assess childhood abuse and household dysfunction before age 18 included eleven questions that are similar to the questions on verbal abuse, physical abuse, sexual abuse, household substance abuse, household mental illness, witnessing domestic violence, incarcerated household member, and parental separation/ divorce before age 18 that were used in the original KaiserCDC study. ${ }^{7,8}$ Verbal abuse was defined as a response of "more than once" to the question "How often did a parent or adult in your home ever swear at you, insult you, or put you down?" Physical abuse was defined as a response of either "once" or "more than once" to the question "How often did your parents or adult in your home ever hit, beat, kick, or physically hurt you in any way? Do not include spanking." Sexual abuse was defined as a response of either "once" or "more than once" to any of the following three questions: "How often did anyone at least 5 years older than you or an adult, ever touch you sexually?", "How often did anyone at least 5 years older than you or an adult try to make you touch them sexually?" or "How often did anyone at least 5 years older than you or an adult force you to have sex?" Living with a household substance abuser was defined as an affirmative response to at least one of two questions about living with anyone who "was a problem drinker or alcoholic" or "used illegal street drugs or abused prescription medications." Household mental illness was defined by an affirmative response to a question about living with 
a household member who "was depressed, mentally ill, or suicidal." Witnessing domestic violence was defined as a response of "once" or "more than once" to the question "How often did your parents or adults in your home ever slap, hit, kick, punch, or beat each other up?" Incarcerated household member was defined by an affirmative response to the question about living with a household member who "served time or was sentenced to serve time in prison, jail, or other correctional facility." Having "parents who were separated or divorced" was defined by an affirmative response to a question asking about that as opposed to answering "no", or "parents not married." The ACEs score was generated by summing the total number of ACEs (range: 0-8). ACEs scores of 5 or more were combined into one category $(\geq 5)$ due to small cell sizes. Therefore, six categories of ACEs score $(0,1,2,3,4$, and $\geq 5)$ were included in these analyses.

\section{COPD}

BRFSS 2011 was the first time the survey included a COPD specific question. COPD was identified among respondents who answered with an affirmative response to the question "Has a doctor, nurse, or other health professional ever told you that you have $[. ..] \mathrm{COPD}$, chronic obstructive pulmonary disease, emphysema, or chronic bronchitis?"

\section{Other variables}

Covariates for these analyses included age group (18-44, $45-54,55-64,65-74$, or $\geq 75$ years), sex (men or women), race/ethnicity (non-Hispanic white; non-Hispanic Black; non-Hispanic Asian; non-Hispanic Native Hawaiian or other Pacific Islander; non-Hispanic American Indian or Alaskan Native; non-Hispanic other race; non-Hispanic multiracial; or Hispanic), marital status (married; previously married; or never married or member of unmarried couple), educational attainment (did not graduate high school, graduated high school or obtained the general equivalent degree, some college or technical school, or graduated college or technical school), employment (employed, unemployed, homemaker/ student, retired, or unable to work), any asthma history, (yes or no), and health insurance coverage (yes or no). Additionally, smoking status (never smoker, former smoker, or current smoker) was considered in this study. Never smokers were respondents who reported smoking fewer than 100 cigarettes during their lifetimes. Former smokers were those respondents who reported ever smoking at least 100 cigarettes but reported smoking "not at all" at the time of the interview. Current cigarette smokers were respondents who reported smoking at least 100 cigarettes during their lifetimes and reported smoking "every day" or "some days" at the time of the interview.

\section{Statistical analysis}

All analyses were conducted using SAS-callable SUDAAN version 11.0 (Research Triangle Institute, Research Triangle Park, NC, USA) to account for the complex sampling design of BRFSS. All estimates were weighted to represent the sampled population. We chose $P<0.10$ as the cutoff for statistical significance of the interactions between sex and ACEs on the relationship of these variables to COPD because this value is the commonly accepted value in the literature for detecting interactions. ${ }^{24}$ All other results were considered significant at $P<0.05$. We have chosen not to make statistical adjustments for multiple comparisons because doing so will increase type II errors. ${ }^{25}$ If observed associations are all the result of chance, type I errors can occur, but type II errors cannot occur. On the other hand, if the observed associations all reflect actual relationships, type II errors can occur, but type I errors cannot. We performed analyses stratified by sex using multivariable log-linear regression. Log-linear regression models were used to estimate the prevalence ratios (PRs) for the likelihood of having COPD and their corresponding 95\% confidence intervals (CIs) and included the following relevant covariates that were expected to impact the dependent variable: age group, race/ethnicity, marital status, educational attainment, employment, asthma history, and health insurance coverage. In addition, we examined the role of smoking status by including it in regression models with ACEs and the other variables and also by examining the relationship between ACEs and COPD by smoking status because smoking is a major risk for COPD.

\section{Results}

The analytic sample was comprised of 26,546 women (50.8\%) and 19,015 men (49.2\%) from five US states. The majority of respondents were non-Hispanic white $(84.8 \%)$ and about a third had at least some college education (33.9\%). More than half $(54.0 \%)$ of respondents were never smokers, $12.7 \%$ reported ever having asthma, and $4.4 \%$ reported having COPD. The distributions of selected characteristics are presented by sex (Table 1). Compared to men, women were significantly $(P<0.05)$ more likely to be aged $\geq 75$ years; to be a non-Hispanic white adult; to be unmarried; to have at least some college education; to be a homemaker/student or retired; to have health insurance coverage; to have a history of asthma; and to be a never smoker. COPD prevalence did 
Table I Number and percent of selected characteristics among adults aged $\geq \mid 18$ years in five states, by sex: Behavioral Risk Factor Surveillance System, $201 \mathrm{I}$

\begin{tabular}{|c|c|c|c|c|c|}
\hline Characteristic & $\mathbf{n}^{\mathbf{a}}$ & $\begin{array}{l}\text { Women } \\
\left.\% \text { (95\% } \mathrm{Cl}^{\mathrm{c}}\right)\end{array}$ & $\mathbf{n}^{\mathbf{a}}$ & $\begin{array}{l}\text { Men } \\
\%{ }^{\mathrm{b}}\left(95 \% \mathrm{Cl}^{\mathrm{c}}\right)\end{array}$ & $\begin{array}{l}P \text {-value for } \\
\text { difference }^{d}\end{array}$ \\
\hline Age group, years & & & & & $<0.001$ \\
\hline $18-44$ & 6,533 & $43.6(42.4,44.8)$ & 5,166 & $46.2(44.9,47.6)$ & \\
\hline $45-54$ & 5,062 & $20.0(19.1,20.9)$ & 3,742 & $20.7(\mid 9.7,21.8)$ & \\
\hline $55-64$ & 6,404 & $16.6(16.0,17.4)$ & 4,760 & I7.I $(16.3,18.0)$ & \\
\hline $65-74$ & 4,599 & $10.2(9.6,10.7)$ & 3,326 & $9.6(9.0,10.2)$ & \\
\hline$\geq 75$ & 3,948 & $9.6(9.1,10.1)$ & 2,021 & $6.3(5.9,6.8)$ & \\
\hline Race/ethnicity & & & & & $<0.01$ \\
\hline White, non-Hispanic & $23,80 I$ & $86.1(85.2,87.1)$ & 17,078 & $83.4(82.2,84.6)$ & \\
\hline Black, non-Hispanic & 559 & $2.9(2.4,3.4)$ & 364 & $3.4(2.8,4.1)$ & \\
\hline Asian & 321 & $3.5(3.0,4.1)$ & 221 & $3.7(3.1,4.4)$ & \\
\hline Native Hawaiian or Pacific Islander & 50 & $0.2(0.1,0.4)$ & 30 & $0.1(0.1,0.2)$ & \\
\hline American Indian or Alaskan Native & 517 & $1.4(1.1,1.7)$ & 345 & $1.8(1.4,2.4)$ & \\
\hline Other race only, non-Hispanic & 147 & $0.3(0.2,0.4)$ & 137 & $0.6(0.4,0.8)$ & \\
\hline Multiracial, non-Hispanic & 420 & I.I $(0.9,1.4)$ & 289 & $1.3(1.0,1.7)$ & \\
\hline Hispanic & 731 & $4.4(3.8,5.1)$ & 551 & $5.6(4.9,6.4)$ & \\
\hline Marital status & & & & & $<0.001$ \\
\hline Married & 14,269 & $55.1(53.9,56.3)$ & 11,696 & $57.0(55.6,58.3)$ & \\
\hline Previously married ${ }^{\mathrm{e}}$ & 8,617 & $22.8(21.9,23.7)$ & 3,236 & $13.4(\mid 2.6,14.3)$ & \\
\hline Never married ${ }^{f}$ & 3,660 & $22.1(21.0,23.4)$ & 3,683 & $29.6(28.3,31.0)$ & \\
\hline Educational attainment & & & & & $<0.001$ \\
\hline Did not graduate high school & 1,375 & $8.6(7.8,9.5)$ & 1,166 & $11.2(10.1,12.3)$ & \\
\hline Graduated high school & 7,158 & $27.6(26.5,28.7)$ & 5,173 & $29.6(28.4,30.9)$ & \\
\hline Some college or technical school & 8,080 & $35.8(34.6,37.0)$ & 5,180 & $31.9(30.6,33.2)$ & \\
\hline Graduated college or technical school & 9,933 & $28.0(27.1,29.0)$ & 7,496 & $27.3(26.2,28.3)$ & \\
\hline Employment & & & & & $<0.001$ \\
\hline Employed & 13,346 & $54.9(53.7,56.1)$ & 11,213 & $65.0(63.7,66.3)$ & \\
\hline Unemployed & $\mathrm{I}, 237$ & $5.9(5.4,6.5)$ & $\mathrm{I}, 177$ & $8.8(7.9,9.7)$ & \\
\hline Homemaker/student & 3,043 & $16.8(15.7,17.9)$ & 448 & $5.2(4.4,6.0)$ & \\
\hline Retired & 7,434 & $17.8(17.1,18.5)$ & 5,297 & $16.6(15.9,17.4)$ & \\
\hline Unable to work & $\mathrm{I}, 486$ & $4.6(4.2,5.1)$ & 880 & $4.4(3.9,5.0)$ & \\
\hline Health insurance coverage & 24,287 & $88.4(87.5,89.3)$ & 16,824 & $84.3(83.2,85.3)$ & $<0.001$ \\
\hline Asthma & 3,778 & $14.6(13.7,15.5)$ & 1,957 & $10.7(9.9,11.6)$ & $<0.001$ \\
\hline Cigarette smoking & & & & & $<0.001$ \\
\hline Never smoker ${ }^{g}$ & 15,099 & $59.0(57.8,60.1)$ & 8,888 & $48.9(47.6,50.3)$ & \\
\hline Former smoker ${ }^{h}$ & 7,661 & $24.2(23.3,25.2)$ & 6,936 & $30.3(29.1,31.5)$ & \\
\hline Current smokeri & 3,786 & $16.8(15.9,17.8)$ & 3,191 & $20.8(19.6,22.0)$ & \\
\hline Chronic obstructive pulmonary disease & 1,660 & $4.9(4.4,5.4)$ & 972 & $4.0(3.5,4.6)$ & 0.02 \\
\hline
\end{tabular}

Notes: ${ }^{a}$ Unweighted number of respondents in Minnesota, Montana, Vermont, Washington, Wisconsin; ${ }^{b}$ weighted percentages; ${ }^{\mathrm{c}} \mathrm{confidence} \mathrm{interval} \mathrm{(Cl);}{ }^{\mathrm{d}}$-values determined using chi-square tests; ${ }^{e}$ previously married includes those divorced, widowed, or separated; fnever married includes those never married or members of unmarried couples; ${ }^{g}$ respondents who smoked fewer than 100 cigarettes during their lifetime were considered never smokers; ${ }^{\mathrm{h}}$ respondents who smoked at least 100 cigarettes and reported smoking not at all at the time of interview were considered former smokers; 'respondents who smoked at least 100 cigarettes and reported smoking every day or on some days at the time of interview were considered current smokers.

not differ significantly by sex in these five states although the prevalence was higher among women.

The overall majority (63.1\%) of respondents reported having had at least one ACE before age 18. Distributions of individual ACEs and ACEs score are presented by sex (Table 2). Compared to women, men were significantly $(P<0.05)$ more likely to report childhood verbal abuse. In contrast, women were significantly $(P<0.05)$ more likely to report childhood sexual abuse; living with a substance abusing household member; living with a mentally ill household member; and to report $\geq 5$ ACEs compared to men.

We assessed the prevalence of COPD by individual ACEs and ACEs score for women and men (Table 3). The unadjusted prevalence of COPD was significantly $(P<0.05)$ higher among women with any individual ACEs than among women without. Additionally, the unadjusted prevalence of COPD was significantly $(P<0.05)$ higher among men with a childhood history of physical abuse, sexual abuse, and household substance abuse. However, the unadjusted 
Table 2 Number and percent of adults aged $\geq 18$ years reporting individual adverse childhood experiences (ACEs) and the distribution of the ACE score in five states, by sex: Behavioral Risk Factor Surveillance System, 20II

\begin{tabular}{|c|c|c|c|c|c|}
\hline Adverse childhood experiences & $\mathbf{n}^{\mathbf{a}}$ & $\begin{array}{l}\text { Women }[\mathrm{N}=26,546] \\
\%^{\mathrm{b}}\left(95 \% \mathrm{Cl}^{\mathrm{c}}\right)\end{array}$ & $\mathbf{n}^{\mathbf{a}}$ & $\begin{array}{l}\text { Men }[N=|9,0| 5] \\
\%{ }^{b}\left(95 \% C^{c}\right)\end{array}$ & $\begin{array}{l}P \text {-value for } \\
\text { difference }^{d}\end{array}$ \\
\hline Verbal abuse & 8,834 & $36.8(35.7,38.0)$ & 6,562 & $38.9(37.6,40.3)$ & 0.02 \\
\hline Physical abuse & 4,121 & $17.3(16.4,18.2)$ & 3,076 & $18.3(17.2,19.5)$ & 0.16 \\
\hline Sexual abuse & 4,407 & $16.7(15.9,17.6)$ & $\mathrm{I}, 28 \mathrm{I}$ & $6.4(5.8,7.1)$ & $<0.001$ \\
\hline Substance abusing household member & 7,650 & $30.5(29.4,31.6)$ & 4,797 & $27.3(26.1,28.6)$ & $<0.01$ \\
\hline Mentally ill household member & 5,032 & $20.6(19.7,21.6)$ & 2,413 & $14.2(13.2,15.3)$ & $<0.001$ \\
\hline Witnessing domestic violence & 4,099 & $17.5(16.6,18.5)$ & 2,723 & $16.4(|5.3| 7.5)$, & 0.11 \\
\hline Incarcerated household member & 1,139 & $6.7(6.0,7.5)$ & 930 & $7.6(6.8,8.5)$ & 0.12 \\
\hline Parental separation/divorce & 5,285 & $24.8(23.8,26.0)$ & 3,790 & $24.7(23.4,25.9)$ & 0.83 \\
\hline ACE score & & & & & $<0.001$ \\
\hline 0 & 10,739 & $36.2(35.1,37.3)$ & 7,912 & $37.7(36.4,39.0)$ & \\
\hline I & 5,884 & $22.9(21.9,24.0)$ & 4,648 & $23.4(22.4,24.7)$ & \\
\hline 2 & 3,464 & $\mid 3.6(|2.8| 4.5)$, & 2,649 & $15.3(14.3,16.3)$ & \\
\hline 3 & 2,360 & $9.5(8.8,10.2)$ & $|, 56|$ & $8.8(8.1,9.6)$ & \\
\hline 4 & 1,664 & $6.5(6.0,7.1)$ & 1,050 & $6.3(5.6,7.0)$ & \\
\hline$\geq 5$ & 2,435 & $11.3(10.5,12.2)$ & 1,195 & $8.4(7.6,9.4)$ & \\
\hline
\end{tabular}

Notes: anweighted sample size; ${ }^{b}$ weighted percentages; ${ }^{c}$ confidence interval (Cl); ${ }^{d}$-values determined using chi-square tests.

prevalence of COPD increased significantly $(P<0.05)$ with an increasing ACEs score only among women.

Because we found a statistically significant interaction $(P<0.10)$ for sex with each ACE and the ACEs score on the likelihood of COPD, we examined sex-specific relationships. We examined the likelihood of having COPD after taking into account the confounding effects of age, asthma, and sociodemographic characteristics (Table 3). We also examined the likelihood of COPD after also adjusting for smoking status as an additional covariate. Adjustment for smoking status attenuated the relationship between most individual ACEs and ACEs score with COPD among both women and men. For women, verbal abuse ( $\mathrm{PR}=1.30,95 \% \mathrm{CI}$ : 1.05 , 1.61), sexual abuse ( $\mathrm{PR}=1.69,95 \% \mathrm{CI}$ : 1.36, 2.10), living with a substance abusing household member $(\mathrm{PR}=1.49$, 95\% CI: 1.23, 1.81), witnessing domestic violence (PR $=1.40,95 \%$ CI: $1.14,1.72)$, and parental separation/divorce during childhood ( $\mathrm{PR}=1.47,95 \% \mathrm{CI}: 1.21,1.80$ ) were all associated with a higher likelihood of COPD compared to women who had not reported those individual ACEs. In contrast, for men, living with an incarcerated household member during childhood ( $\mathrm{PR}=0.49,95 \% \mathrm{CI}$ : $0.31,0.77$ ) was associated with a lower likelihood of COPD compared to men who had not reported that individual ACEs. Reporting $\geq 5$ ACEs ( $P R=2.08,95 \%$ CI: $1.55,2.80$ ) was associated with a higher likelihood of COPD compared to reporting no ACEs only among women.

We assessed the prevalence of COPD by individual ACEs and ACE score for women and men who had never smoked (Table 4). As expected, the prevalence of COPD among persons who had never smoked was low. The unadjusted prevalence of COPD was significantly $(P<0.05)$ higher among women who had never smoked but had a childhood history of physical abuse, sexual abuse, and household substance abuse. We also examined the likelihood of having COPD among women and men who were never smokers (Table 4). For women who were never smokers, there was still a higher likelihood of COPD associated with physical abuse ( $\mathrm{PR}=1.81,95 \% \mathrm{CI}: 1.28,2.58$ ), sexual abuse (PR $=2.18,95 \%$ CI: 1.46, 3.24), and living with a substance abusing household member during childhood ( $\mathrm{PR}=1.70$, $95 \%$ CI: $1.18,2.43$ ). Reporting $\geq 5$ ACEs (PR $=2.08,95 \%$ CI: $1.25,3.46)$ remained associated with a higher likelihood of COPD compared to reporting no ACEs among women who were never smokers.

We assessed the likelihood of having COPD among women and men who were former smokers (Table 5). For women who were former smokers, there was a higher likelihood of COPD associated with verbal abuse ( $\mathrm{PR}=1.55,95 \% \mathrm{CI}: 1.17,2.05$ ), sexual abuse ( $\mathrm{PR}=2.06,95 \% \mathrm{CI}$ : 1.55, 2.72), living with a substance abusing household member during childhood ( $\mathrm{PR}=1.75,95 \%$ CI: 1.38, 2.22), witnessing domestic violence ( $\mathrm{PR}=1.85,95 \% \mathrm{CI}: 1.43,2.39$ ), and parental separation/ divorce ( $\mathrm{PR}=1.80,95 \% \mathrm{CI}: 1.39,2.34)$. Reporting $\geq 5$ ACEs $(\mathrm{PR}=3.04,95 \% \mathrm{CI}: 2.16,4.29)$ remained associated with a higher likelihood of COPD compared to reporting no ACEs among women who were former smokers. For men who were former smokers, there was a higher likelihood of COPD 
Table 3 Prevalence and the likelihood of chronic obstructive pulmonary disease with individual adverse childhood experiences (ACEs) and the ACE score among adults aged $\geq 18$ years in five states, by sex: Behavioral Risk Factor Surveillance System, 201 I

\begin{tabular}{|c|c|c|c|c|c|c|}
\hline \multirow{2}{*}{$\begin{array}{l}\text { Adverse childhood } \\
\text { experiences }\end{array}$} & \multicolumn{3}{|l|}{ Women } & \multicolumn{3}{|l|}{ Men } \\
\hline & $\begin{array}{l}\text { Unadjusted \% } \\
\left(95 \% \mathrm{Cl}^{\mathrm{a}}\right)\end{array}$ & $\begin{array}{l}{[N=26,546]} \\
P^{b}\left(95 \% C l^{a}\right)\end{array}$ & $\operatorname{PR}^{\mathrm{c}}\left(95 \% \mathrm{Cl}^{\mathrm{a}}\right)$ & $\begin{array}{l}\text { Unadjusted \% } \\
\left(95 \% \mathrm{Cl}^{\mathrm{a}}\right)\end{array}$ & $\begin{array}{l}{[N=|9,0| 5]} \\
P^{b}\left(95 \% C^{a}\right)\end{array}$ & $\operatorname{PR}^{c}\left(95 \% \mathrm{Cl}^{\mathrm{a}}\right)$ \\
\hline \multicolumn{7}{|l|}{ Verbal abuse } \\
\hline No & $4.0(3.5,4.6)$ & 1.00 (referent) & $\mathrm{I} .00$ (referent) & $4.2(3.5,5.0)$ & $\mathrm{I} .00$ (referent) & 1.00 (referent) \\
\hline Yes & $6.4(5.5,7.4)$ & $1.45(I .15,1.8 \mathrm{I})$ & $1.30(1.05,1.61)$ & $3.7(3.1,4.5)$ & $\mathrm{I} .03(0.8 \mathrm{I}, \mathrm{I} .3 \mathrm{I})$ & $0.97(0.76,1.24)$ \\
\hline \multicolumn{7}{|l|}{ Physical abuse } \\
\hline No & $4.0(3.6,4.5)$ & 1.00 (referent) & $\mathrm{I} .00$ (referent) & $3.7(3.1,4.3)$ & $\mathrm{I} .00$ (referent) & $\mathrm{I} .00$ (referent) \\
\hline Yes & $8.9(7.3,10.7)$ & $1.4 \mathrm{I}(\mathrm{I} .14, \mathrm{I} .75)$ & $1.23(0.99,1.52)$ & $5.6(4.4,7.0)$ & $1.30(0.98,1.73)$ & $1.18(0.89,1.57)$ \\
\hline \multicolumn{7}{|l|}{ Sexual abuse } \\
\hline No & $3.7(3.3,4.2)$ & $\mathrm{I} .00$ (referent) & 1.00 (referent) & $3.8(3.3,4.4)$ & $\mathrm{I} .00$ (referent) & $\mathrm{I} .00$ (referent) \\
\hline Yes & $10.6(8.8,12.6)$ & $1.93(I .55,2.4 I)$ & $1.69(1.36,2.11)$ & $6.9(4.9,9.6)$ & $1.09(0.77,1.54)$ & $0.99(0.70,1.39)$ \\
\hline \multicolumn{7}{|c|}{ Substance abusing household member } \\
\hline No & $3.6(3.1,4.1)$ & I.00 (referent) & $\mathrm{I} .00$ (referent) & $3.7(3.1,4.4)$ & $\mathrm{I} .00$ (referent) & 1.00 (referent) \\
\hline Yes & $7.7(6.6,9.0)$ & $1.76(1.49,2.15)$ & $1.49(1.23,1.81)$ & $4.9(4.0,5.9)$ & $1.36(1.05,1.76)$ & $1.23(0.95,1.59)$ \\
\hline \multicolumn{7}{|c|}{ Mentally ill household member } \\
\hline No & $4.4(3.9,4.9)$ & 1.00 (referent) & $\mathrm{I} .00$ (referent) & $4.0(3.5,4.6)$ & $\mathrm{I} .00$ (referent) & 1.00 (referent) \\
\hline Yes & $6.8(5.7,8.2)$ & $1.26(0.99,1.61)$ & $1.13(0.89,1.44)$ & $4.1(3.0,5.6)$ & $1.18(0.82,1.68)$ & $1.07(0.76, \mathrm{I} .50)$ \\
\hline \multicolumn{7}{|c|}{ Witnessing domestic violence } \\
\hline No & $4.0(3.6,4.5)$ & $\mathrm{I} .00$ (referent) & 1.00 (referent) & $4.0(3.5,4.7)$ & $\mathrm{I} .00$ (referent) & $\mathrm{I} .00$ (referent) \\
\hline Yes & $8.8(7.1,10.7)$ & $1.65(1.34,2.03)$ & $1.40(1.14,1.72)$ & $4.0(3.1,5.2)$ & $0.92(0.69,1.21)$ & $0.8 \mathrm{I}(0.6 \mathrm{I}, \mathrm{I} .08)$ \\
\hline \multicolumn{7}{|c|}{ Incarcerated household member } \\
\hline No & $4.6(4.2,5.2)$ & 1.00 (referent) & $\mathrm{I} .00$ (referent) & $4.1(3.6,4.7)$ & $\mathrm{I} .00$ (referent) & 1.00 (referent) \\
\hline Yes & $8.0(5.9,10.8)$ & $1.30(0.95,1.78)$ & $\mathrm{I} .10(0.80, \mathrm{I} .5 \mathrm{I})$ & $2.7(1.9,3.9)$ & $0.59(0.38,0.91)$ & $0.49(0.3 \mathrm{I}, 0.77)$ \\
\hline \multicolumn{7}{|c|}{ Parental separation/divorce } \\
\hline No & $4.2(3.8,4.6)$ & 1.00 (referent) & $\mathrm{I} .00$ (referent) & $4.1(3.6,4.7)$ & $\mathrm{I} .00$ (referent) & 1.00 (referent) \\
\hline Yes & $7.0(5.6,8.7)$ & $1.76(1.43,2.17)$ & $1.47(1.20,1.79)$ & $3.7(2.7,5.2)$ & $1.05(0.75,1.46)$ & $0.93(0.68,1.26)$ \\
\hline \multicolumn{7}{|l|}{ ACE score } \\
\hline 0 & $3.0(2.6,3.6)$ & 1.00 (referent) & $\mathrm{I} .00$ (referent) & $3.8(3.0,4.7)$ & 1.00 (referent) & 1.00 (referent) \\
\hline 1 & $4.0(3.0,5.4)$ & $1.40(1.03,1.89)$ & $1.19(0.90,1.56)$ & $4.2(3.0,5.8)$ & $1.18(0.84,1.65)$ & $\mathrm{I} .12(0.8 \mathrm{I}, \mathrm{I} .55)$ \\
\hline 2 & $4.9(3.8,6.2)$ & $1.55(1.17,2.06)$ & $1.31(1.00,1.73)$ & $3.2(2.3,4.5)$ & $1.00(0.69,1.47)$ & $0.91(0.63,1.33)$ \\
\hline 3 & $5.3(4.2,6.7)$ & $1.65(1.17,2.33)$ & $1.32(0.92,1.87)$ & $5.7(4.1,7.8)$ & $1.6 I(I .12,2.31)$ & $\mathrm{I} .48(\mathrm{I} .04,2.1 \mathrm{I})$ \\
\hline 4 & $6.1(4.5,8.2)$ & $1.91(1.37,2.67)$ & $1.53(1.10,2.14)$ & $4.2(2.7,6.5)$ & $\mathrm{I} .23(0.75,2.0 \mathrm{I})$ & $1.08(0.67,1.77)$ \\
\hline$\geq 5$ & $11.5(9.1,14.3)$ & $2.86(2.13,3.85)$ & $2.08(1.55,2.79)$ & $4.3(3.1,5.8)$ & I.II $(0.72$, I.72) & $0.85(0.54,1.33)$ \\
\hline
\end{tabular}

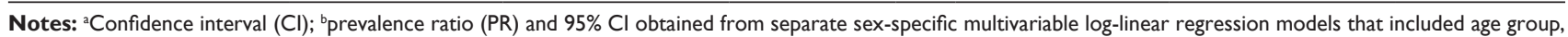
race/ethnicity, marital status, educational attainment, employment, asthma history, and health insurance coverage; ${ }^{\mathrm{PR}}$ and $95 \% \mathrm{Cl}$ obtained from separate sex-specific multivariable log-linear regression models that included the same covariates as previous models with the addition of smoking status.

associated with physical abuse ( $\mathrm{PR}=1.59,95 \% \mathrm{CI}: 1.08,2.34)$ and living with a substance abusing household member during childhood ( $\mathrm{PR}=1.44,95 \%$ CI: 1.01, 2.05).

We assessed the likelihood of having COPD among women and men who were current smokers (Table 6). For women who were current smokers, there was a higher likelihood of COPD associated with parental separation/divorce (PR $=1.37,95 \%$ CI: 1.03, 1.83).

We ran sensitivity analyses including depression as a potential covariate because depression might result from ACEs and potentially operate through smoking. Depression was identified among respondents who answered with an affirmative response to the question "Has a doctor, nurse, or other health professional ever told you that you have $[\ldots]$ a depressive disorder (including depression, major depression, dysthymia, or minor depression)?"'The inclusion of depression reduced the analytic sample to 26,451 women and 18,946 men with complete records. Women were significantly more likely to report depression compared to men $(21.3 \%$ versus $12.8 \%$; $P<0.05$ ). Reporting $\geq 5$ ACEs remained associated with a higher likelihood of COPD compared to reporting no ACEs among all women ( $\mathrm{PR}=1.84,95 \% \mathrm{CI}$ : $1.38,2.45)$, women who were never smokers ( $\mathrm{PR}=1.78,95 \% \mathrm{CI}: 1.06,2.98)$, and women who were former smokers ( $\mathrm{PR}=2.75,95 \% \mathrm{CI}$ : 1.94 , $3.91)$ in the stratified multivariable log-linear regressions after also adjusting for depression.

\section{Discussion}

This analysis of representative, population-based data in five states indicates that experiences of childhood 
Table 4 Prevalence and the likelihood of chronic obstructive pulmonary disease associated with individual adverse childhood experiences (ACEs) and the ACE score among adults aged $\geq 18$ years who were never smokers in five states, by sex: Behavioral Risk Factor Surveillance System, 20I I

\begin{tabular}{|c|c|c|c|c|}
\hline \multirow{2}{*}{$\begin{array}{l}\text { Adverse childhood } \\
\text { experiences }\end{array}$} & \multicolumn{2}{|c|}{ Women $[\mathrm{N}=15,099]$} & \multicolumn{2}{|l|}{ Men $[\mathrm{N}=\mathbf{8 , 8 8 8}]$} \\
\hline & $\begin{array}{l}\text { Unadjusted \% } \\
\left(95 \% \mathrm{Cl}^{\mathrm{a}}\right)\end{array}$ & $\mathbf{P R}^{\mathrm{b}}\left(95 \% \mathrm{Cl}^{\mathrm{a}}\right)$ & $\begin{array}{l}\text { Unadjusted \% } \\
\left(95 \% \mathrm{Cl}^{\mathrm{a}}\right)\end{array}$ & $\mathbf{P R}^{\mathrm{b}}\left(95 \% \mathrm{Cl}^{\mathrm{a}}\right)$ \\
\hline \multicolumn{5}{|l|}{ Verbal abuse } \\
\hline No & $1.4(I . I, I .7)$ & 1.00 (referent) & $1.5(1.1,2.1)$ & 1.00 (referent) \\
\hline Yes & $1.7(1.4,2.2)$ & $1.34(0.93,1.91)$ & $1.2(0.7,1.9)$ & $1.02(0.57, \mathrm{I} .8 \mathrm{I})$ \\
\hline \multicolumn{5}{|l|}{ Physical abuse } \\
\hline No & $1.3(1.0,1.5)$ & $\mathrm{I} .00$ (referent) & $1.5(1.1,2.0)$ & 1.00 (referent) \\
\hline Yes & $3.0(2.3,4.0)$ & $1.81(1.28,2.58)$ & $0.9(0.4,1.9)$ & $0.56(0.25,1.26)$ \\
\hline \multicolumn{5}{|l|}{ Sexual abuse } \\
\hline No & $1.2(1.0,1.5)$ & $\mathrm{I} .00$ (referent) & $1.4(1.0,1.9)$ & 1.00 (referent) \\
\hline Yes & $3.5(2.5,4.8)$ & $2.18(1.46,3.24)$ & I.I $(0.4,2.8)$ & $0.56(0.19,1.64)$ \\
\hline \multicolumn{5}{|c|}{ Substance abusing household member } \\
\hline No & I.3 (I.0, I.6) & 1.00 (referent) & $1.3(0.9,1.9)$ & $\mathrm{I} .00$ (referent) \\
\hline Yes & $2.1(1.6,2.8)$ & $1.70(1.18,2.43)$ & $1.7(1.0,2.6)$ & $1.36(0.76,2.42)$ \\
\hline \multicolumn{5}{|c|}{ Mentally ill household member } \\
\hline No & $1.4(1.1,1.7)$ & $\mathrm{I} .00$ (referent) & $1.4(1.0,1.9)$ & 1.00 (referent) \\
\hline Yes & $2.0(1.4,2.9)$ & $\mathrm{I} .47(0.93,2.33)$ & $1.2(0.7,2.2)$ & $0.97(0.47,1.96)$ \\
\hline \multicolumn{5}{|c|}{ Witnessing domestic violence } \\
\hline No & $1.4(1.2,1.7)$ & $\mathrm{I} .00$ (referent) & $1.5(1.1,2.0)$ & $\mathrm{I} .00$ (referent) \\
\hline Yes & $2.0(1.4,2.7)$ & $1.37(0.94,1.98)$ & $1.0(0.5,2.1)$ & $0.78(0.32,1.90)$ \\
\hline \multicolumn{5}{|c|}{ Incarcerated household member } \\
\hline No & I.5 (I.2, I.8) & 1.00 (referent) & $1.4(1.1,1.9)$ & $\mathrm{I} .00$ (referent) \\
\hline Yes & $1.6(0.8,3.1)$ & $\mathrm{I} .08(0.52,2.24)$ & $0.6(0.3,1.4)$ & $0.48(0.18,1.34)$ \\
\hline \multicolumn{5}{|c|}{ Parental separation/divorce } \\
\hline No & I.5 (I.3, I.8) & $\mathrm{I} .00$ (referent) & $1.4(1.0,1.9)$ & $\mathrm{I} .00$ (referent) \\
\hline Yes & $1.3(1.0,1.9)$ & $1.02(0.68,1.53)$ & $1.6(0.9,2.8)$ & $1.66(0.84,3.29)$ \\
\hline \multicolumn{5}{|l|}{ ACE score } \\
\hline 0 & $1.5(1.1,1.9)$ & 1.00 (referent) & $1.5(1.0,2.4)$ & $\mathrm{I} .00$ (referent) \\
\hline I & $0.8(0.6,1.2)$ & $0.72(0.46,1.12)$ & $1.5(0.8,2.7)$ & $0.99(0.54,1.83)$ \\
\hline 2 & $1.2(0.8,1.9)$ & $0.99(0.57,1.73)$ & $1.0(0.8,1.8)$ & $0.78(0.35,1.75)$ \\
\hline 3 & $2.3(1.2,4.1)$ & $1.85(0.91,3.75)$ & $1.2(0.6,2.4)$ & $0.89(0.37,2.16)$ \\
\hline 4 & $2.0(1.2,3.2)$ & $\mathrm{I} .63(0.95,2.82)$ & $1.5(0.6,3.9)$ & $\mathrm{I} .07(0.37,3 . \mathrm{II})$ \\
\hline$\geq 5$ & $3.1(2.1,4.5)$ & $2.08(1.25,3.46)$ & $1.3(0.4,4.6)$ & $1.29(0.3 \mathrm{I}, 5.26)$ \\
\hline
\end{tabular}

Notes: a Confidence interval $(\mathrm{Cl}) ;{ }^{b}$ prevalence ratio (PR) and $95 \% \mathrm{Cl}$ obtained from separate sex-specific multivariable log-linear regression models that included age group, race/ethnicity, marital status, educational attainment, employment, asthma history, and health insurance coverage.

maltreatment and household dysfunction are related to COPD; however, this association varies for women and men. For women, after taking into account all the covariates including smoking and other covariates, verbal abuse, sexual abuse, living with a substance abusing household member, witnessing domestic violence, parental separation/ divorce, and the number of ACEs experienced before age 18 were all associated with a higher likelihood of COPD. In contrast, for men, living with an incarcerated household member was associated with a lower likelihood of COPD. Moreover, for women who were never smokers, physical abuse, sexual abuse, and living with a substance abusing household member before age 18 were also associated with a higher likelihood of COPD.
While previous epidemiologic studies have reported on the relationship between ACEs and COPD, ${ }^{7,11}$ this study is notable because it is the first to suggest that sex modifies this relationship and it is also the largest study so far. The findings from the original ACEs study of health maintenance organization members in California mostly agree with our observations for the relationships between individual ACEs and the number of ACEs with COPD among women. In that study there was a graded relationship between the ACEs score with COPD prevalence, hospitalizations for COPD, and rates of prescriptions to treat COPD and the authors also observed that these relationships were only modestly attenuated after the adjustment for smoking status. ${ }^{11}$ In another analysis of the original ACEs study, a graded relationship between the ACEs score and smoking 
Table 5 Prevalence and the likelihood of chronic obstructive pulmonary disease associated with individual adverse childhood experiences (ACEs) and the ACE score among adults aged $\geq 18$ years who were former smokers in five states, by sex: Behavioral Risk Factor Surveillance System, 20I I

\begin{tabular}{|c|c|c|c|c|}
\hline \multirow{2}{*}{$\begin{array}{l}\text { Adverse childhood } \\
\text { experiences }\end{array}$} & \multicolumn{2}{|c|}{ Women $[\mathrm{N}=7,66 \mathrm{I}]$} & \multicolumn{2}{|c|}{ Men $[\mathrm{N}=6,936]$} \\
\hline & $\begin{array}{l}\text { Unadjusted \% } \\
\left(95 \% \mathrm{Cl}^{\mathrm{a}}\right)\end{array}$ & $\operatorname{PR}^{\mathrm{b}}\left(95 \% \mathrm{Cl}^{\mathrm{a}}\right)$ & $\begin{array}{l}\text { Unadjusted \% } \\
\left(95 \% \mathrm{Cl}^{2}\right)\end{array}$ & $\operatorname{PR}^{\mathrm{b}}\left(95 \% \mathrm{Cl}^{\mathrm{a}}\right)$ \\
\hline \multicolumn{5}{|l|}{ Verbal abuse } \\
\hline No & $6.9(5.9,8.0)$ & $\mathrm{I} .00$ (referent) & $6.1(4.9,7.5)$ & $\mathrm{I} .00$ (referent) \\
\hline Yes & $10.7(8.3,13.8)$ & $1.55(1.17,2.05)$ & $6.2(4.8,8.0)$ & $1.20(0.87,1.65)$ \\
\hline \multicolumn{5}{|l|}{ Physical abuse } \\
\hline No & $7.2(6.1,8.4)$ & $\mathrm{I} .00$ (referent) & $5.5(4.6,6.6)$ & $\mathrm{I} .00$ (referent) \\
\hline Yes & $13.2(9.4,18.3)$ & $1.30(0.96,1.76)$ & $8.6(6.2,11.8)$ & $1.59(1.08,2.34)$ \\
\hline \multicolumn{5}{|l|}{ Sexual abuse } \\
\hline No & $6.5(5.6,7.5)$ & I.00 (referent) & $6.1(5.1,7.2)$ & $\mathrm{I} .00$ (referent) \\
\hline Yes & I5.7 (I I.6, 20.9) & $2.06(1.55,2.72)$ & $6.4(3.9,10.4)$ & $0.78(0.46,1.32)$ \\
\hline \multicolumn{5}{|c|}{ Substance abusing household member } \\
\hline No & $6.1(5.2,7.1)$ & $\mathrm{I} .00$ (referent) & $5.6(4.6,6.9)$ & $\mathrm{I} .00$ (referent) \\
\hline Yes & I2.5 (9.8, I5.9) & $1.75(1.38,2.22)$ & $7.2(5.4,9.6)$ & $\mathrm{I} .44(\mathrm{I} .0 \mathrm{I}, 2.05)$ \\
\hline \multicolumn{5}{|c|}{ Mentally ill household member } \\
\hline No & $7.6(6.4,9.0)$ & $\mathrm{I} .00$ (referent) & $6.4(5.4,7.6)$ & 1.00 (referent) \\
\hline Yes & II.3 (8.3, I5.I) & $1.30(0.90,1.87)$ & $4.3(2.9,6.5)$ & $0.74(0.48, I .14)$ \\
\hline \multicolumn{5}{|c|}{ Witnessing domestic violence } \\
\hline No & $6.8(5.9,7.8)$ & $\mathrm{I} .00$ (referent) & $6.2(5.2,7.4)$ & $\mathrm{I} .00$ (referent) \\
\hline Yes & $14.7(10.5,20.1)$ & $1.85(1.43,2.39)$ & $5.7(3.9,8.3)$ & $0.93(0.61,1.43)$ \\
\hline \multicolumn{5}{|c|}{ Incarcerated household member } \\
\hline No & $8.4(7.1,9.8)$ & $\mathrm{I} .00$ (referent) & $6.3(5.3,7.4)$ & $\mathrm{I} .00$ (referent) \\
\hline Yes & $9.2(5.7,14.5)$ & $1.20(0.77,1.87)$ & $3.6(1.9,6.7)$ & $0.55(0.26,1.17)$ \\
\hline \multicolumn{5}{|c|}{ Parental separation/divorce } \\
\hline No & $7.5(6.4,8.6)$ & 1.00 (referent) & $6.7(5.6,8.0)$ & 1.00 (referent) \\
\hline Yes & II.2(7.9, I5.6) & $\mathrm{I} .80(1.39,2.34)$ & $4.0(2.9,5.7)$ & $0.68(0.45,1.03)$ \\
\hline \multicolumn{5}{|l|}{ ACE score } \\
\hline 0 & $5.6(4.6,6.9)$ & I.00 (referent) & $6.1(4.6,8.0)$ & $\mathrm{I} .00$ (referent) \\
\hline 1 & $6.4(4.7,8.6)$ & $1.20(0.89,1.60)$ & $5.7(4.1,7.8)$ & $0.89(0.61,1.29)$ \\
\hline 2 & $8.4(6.1,11.5)$ & $1.58(1.11,2.25)$ & $4.9(3.2,7.6)$ & $0.89(0.53,1.50)$ \\
\hline 3 & $7.0(4.9,9.9)$ & I.II (0.63, I.98) & $10.1(6.6,15.2)$ & $1.90(1.17,3.08)$ \\
\hline 4 & II.7 (7.I, I8.7) & $2.28(1.39,3.76)$ & $6.9(3.7,12.4)$ & I.4I $(0.73,2.7 I)$ \\
\hline$\geq 5$ & $17.6(\mid 1.9,25.2)$ & $3.04(2.16,4.29)$ & $4.1(2.5,6.7)$ & $0.67(0.33,1.35)$ \\
\hline
\end{tabular}

Notes: a Confidence interval (Cl); ${ }^{b}$ prevalence ratio (PR) and $95 \% \mathrm{Cl}$ obtained from separate sex-specific multivariable log-linear regression models that included age group, race/ethnicity, marital status, educational attainment, employment, asthma history, and health insurance coverage.

during adolescence and adulthood was observed. ${ }^{11}$ Additionally, in a study of the 2009 BRFSS, a graded association between ACEs score and smoking status was observed. ${ }^{12}$ The growing evidence on the relationship between ACEs with COPD and smoking and the relatively high prevalence of ACEs highlight the continuing need for programs and policies that decrease exposure to dysfunction and maltreatment and increase safe, stable, nurturing relationships and environments for children and families in addition to programs and policies that prevent smoking initiation and support cessation. ${ }^{26}$

The pathways by which ACEs influence COPD cannot be determined from this study alone; other unexamined factors contribute. In particular, stressful and traumatic experiences of maltreatment and household dysfunction during childhood are hypothesized to lead to chronic diseases such as COPD in adulthood through behaviors such as smoking and also emotional and psychophysiological responses, including hyperactivity of the hypothalamic-pituitary-adrenal axis; 6,9 this may help explain why the relationship between ACEs and COPD persisted among women who were never smokers and the rather modest attenuation of the relationship between ACEs and COPD even after the adjustment for smoking and depression. ${ }^{27}$ Of note, in a study of the 2010 BRFSS, depressive symptoms were shown to only partially explain some of the relationship between ACEs and smoking. ${ }^{28}$ Similarly, in our analyses, the inclusion of depression did very little to explain the relationship between ACEs and COPD. Nonetheless, a number of questions regarding this relationship 
Table 6 Prevalence and the likelihood of chronic obstructive pulmonary disease associated with individual adverse childhood experiences (ACEs) and the ACE score among adults aged $\geq 18$ years who were current smokers in five states, by sex: Behavioral Risk Factor Surveillance System, 20I I

\begin{tabular}{|c|c|c|c|c|}
\hline \multirow{2}{*}{$\begin{array}{l}\text { Adverse childhood } \\
\text { experiences }\end{array}$} & \multicolumn{2}{|c|}{ Women $[N=3,786]$} & \multicolumn{2}{|l|}{ Men $[N=3, \mid 91]$} \\
\hline & $\begin{array}{l}\text { Unadjusted \% } \\
\left(95 \% \mathrm{Cl}^{\mathrm{a}}\right)\end{array}$ & $\mathbf{P R}^{\mathrm{b}}\left(95 \% \mathrm{Cl}^{\mathrm{a}}\right)$ & $\begin{array}{l}\text { Unadjusted \% } \\
\left(95 \% \mathrm{Cl}^{\mathrm{a}}\right)\end{array}$ & $\mathbf{P R}^{\mathrm{b}}\left(95 \% \mathrm{Cl}^{\mathrm{a}}\right)$ \\
\hline \multicolumn{5}{|l|}{ Verbal abuse } \\
\hline No & II.7 (8.8, I5.5) & $\mathrm{I} .00$ (referent) & $9.1(6.3,13.0)$ & $\mathrm{I} .00$ (referent) \\
\hline Yes & II $.6(9.5,14.1)$ & $1.07(0.77, \mathrm{I} .49)$ & $5.2(3.9,7.0)$ & $0.85(0.58,1.25)$ \\
\hline \multicolumn{5}{|l|}{ Physical abuse } \\
\hline No & $10.8(8.6,13.5)$ & 1.00 (referent) & $6.9(4.9,9.6)$ & 1.00 (referent) \\
\hline Yes & $13.9(\mid 0.7,17.9)$ & $1.02(0.75,1.40)$ & $7.8(5.5,11.0)$ & I.I3 $(0.73,1.75)$ \\
\hline \multicolumn{5}{|l|}{ Sexual abuse } \\
\hline No & $10.1(8.0,12.7)$ & 1.00 (referent) & $6.4(4.8,8.6)$ & 1.00 (referent) \\
\hline Yes & $15.9(\mid 2.3,20.3)$ & $1.24(0.90,1.70)$ & |4.| (8.8, 21.7) & $1.34(0.8 \mathrm{I}, 2.20)$ \\
\hline \multicolumn{5}{|c|}{ Substance abusing household member } \\
\hline No & II.0 (8.3, 14.4) & 1.00 (referent) & $7.7(5.4,10.9)$ & 1.00 (referent) \\
\hline Yes & I2.5 (I0.I, I5.3) & $1.15(0.84,1.56)$ & $6.2(4.4,8.7)$ & $1.00(0.67,1.49)$ \\
\hline \multicolumn{5}{|c|}{ Mentally ill household member } \\
\hline No & II.4 (9.I, I4.2) & 1.00 (referent) & $6.9(5.1,9.3)$ & $\mathrm{I} .00$ (referent) \\
\hline Yes & $12.2(9.3,15.9)$ & $0.97(0.7 \mathrm{I}, \mathrm{I} .33)$ & $8.1(5.1,12.8)$ & $1.43(0.89,2.30)$ \\
\hline \multicolumn{5}{|c|}{ Witnessing domestic violence } \\
\hline No & $10.9(8.7,13.6)$ & $\mathrm{I} .00$ (referent) & $7.6(5.6,10.3)$ & 1.00 (referent) \\
\hline Yes & $13.5(\mid 0.2,17.6)$ & $1.08(0.79,1.48)$ & $5.7(3.9,8.4)$ & $0.75(0.49,1.15)$ \\
\hline \multicolumn{5}{|c|}{ Incarcerated household member } \\
\hline No & II.I $(9.2,13.5)$ & 1.00 (referent) & $7.8(5.9,10.2)$ & 1.00 (referent) \\
\hline Yes & $14.8(9.7,21.9)$ & $1.21(0.78,1.86)$ & $3.5(2.1,5.7)$ & $0.42(0.22,0.8 \mathrm{I})$ \\
\hline \multicolumn{5}{|c|}{ Parental separation/divorce } \\
\hline No & $10.9(9.0,13.2)$ & $\mathrm{I} .00$ (referent) & $7.8(5.9,10.3)$ & $\mathrm{I} .00$ (referent) \\
\hline Yes & $12.7(9.3,17.1)$ & $1.37(1.03,1.83)$ & $6.1(3.5,10.2)$ & $0.92(0.59,1.42)$ \\
\hline \multicolumn{5}{|l|}{ ACE score } \\
\hline 0 & $9.2(6.3,13.2)$ & 1.00 (referent) & $8.1(5.1,12.6)$ & 1.00 (referent) \\
\hline 1 & | $3.3(8.0,2 \mid .4)$ & $\mathrm{I} .42(0.84,2.4 \mathrm{I})$ & $9.3(4.9,17.2)$ & $1.45(0.85,2.40)$ \\
\hline 2 & $10.6(6.7,16.4)$ & $1.26(0.75,2.10)$ & $5.6(3.1,10.0)$ & $1.07(0.58,1.98)$ \\
\hline 3 & $9.8(6.8,13.9)$ & $1.32(0.83,2.1 \mathrm{I})$ & $6.9(3.8,12.3)$ & I.37 $(0.77,2.43)$ \\
\hline 4 & $8.2(5.5,11.9)$ & $0.95(0.56,1.60)$ & $4.4(1.9,9.6)$ & $0.86(0.4 \mathrm{I}, \mathrm{I} .80)$ \\
\hline$\geq 5$ & I5.5 (II.6, 20.4) & $\mathrm{I} .55(0.96,2.5 \mathrm{I})$ & $6.4(4.2,9.6)$ & $\mathrm{I} .02(0.57, \mathrm{I} .8 \mathrm{I})$ \\
\hline
\end{tabular}

Notes: ${ }^{2}$ Confidence interval (Cl); ${ }^{b}$ prevalence ratio (PR) and $95 \% \mathrm{Cl}$ obtained from separate sex-specific multivariable log-linear regression models that included age group, race/ethnicity, marital status, educational attainment, employment, asthma history, and health insurance coverage.

remain unanswered. Future studies investigating this relationship ought to consider the following: 1) are women particularly traumatized by particular ACEs? 2) are women more likely to misreport their smoking status? 3) are there other risks (such as occupational and residential exposures) that contribute to COPD among women?

An explanation for the striking sex differences observed in this study is not readily available. Our results, however, are similar to a previous study on ACEs and smoking that found verbal abuse and sexual abuse during childhood were more strongly related to smoking among women compared to men. ${ }^{13}$ Likewise, female smokers may be more susceptible to COPD compared to male smokers. ${ }^{29}$ A previous systematic review and meta-analysis of longitudinal studies indicates that the lung function in female smokers declines faster than in male smokers. ${ }^{22}$

Our findings should be interpreted in light of several limitations. First, the self-reporting of retrospective ACEs and other variables such as smoking status and COPD was not validated and might be subject to recall and other response biases. ${ }^{30}$ Second, BRFSS data are collected via telephone survey and may not be representative of adults in households without landlines and cell phones. Third, this study only assessed cigarette smoking and not other forms of combustible tobacco use, marijuana use, or secondhand smoke exposure. Finally, as the data were collected from adults in five states, the generalizability of our findings may be limited. 


\section{Conclusion}

In conclusion, the findings of this study suggest that experiences of childhood maltreatment and household dysfunction are more highly related to COPD in women than in men. That is, more significant associations between ACEs and COPD were found for women than for men; different associations were also apparent when results were stratified by smoking status. Insofar as programs and policies that promote safe, stable, and nurturing childhood relationships and environments reduce the burden of ACEs, they might also have the potential to minimize the health consequence of COPD for women. Additionally, programs and policies that address the underlying problems caused by experiences of childhood maltreatment and household dysfunction may prove more effective than traditional smoking prevention and cessation strategies alone. Further research examining sex differences in the relationship between ACEs and chronic diseases in adulthood is warranted.

\section{Disclosure}

The findings and conclusions in this report are those of the authors and do not necessarily represent the official position of the Centers for Disease Control and Prevention. The authors have no conflicts of interest to report.

\section{References}

1. Ford ES, Croft JB, Mannino DM, Wheaton AG, Zhang X, Giles WH. COPD surveillance - United States, 1999-2011. Chest. 2013;144(1): 284-305.

2. Centers for Disease Control and Prevention (CDC). Smokingattributable mortality, years of potential life lost, and productivity losses - United States, 2000-2004. MMWR Morb Mortal Wkly Rep. 2008;57(45):1226-1228.

3. Centers for Disease Control and Prevention (CDC). Chronic obstructive pulmonary disease among adults - United States, 2011. MMWR Morb Mortal Wkly Rep. 2012;61(46):938-943.

4. Ben-Shlomo Y, Kuh D. A life course approach to chronic disease epidemiology: conceptual models, empirical challenges and interdisciplinary perspectives. Int J Epidemiol. 2002;31(2):285-293.

5. Reid DD. The beginnings of bronchitis. Proc R Soc Med. 1969;62(4): 311-316.

6. Anda RF, Butchart A, Felitti VJ, Brown DW. Building a framework for global surveillance of the public health implications of adverse childhood experiences. Am J Prev Med. 2010;39(1):93-98.

7. Felitti VJ, Anda RF, Nordenberg D, et al. Relationship of childhood abuse and household dysfunction to many of the leading causes of death in adults. The Adverse Childhood Experiences (ACE) Study. Am J Prev Med. 1998;14(4):245-258.

8. Centers for Disease Control and Prevention (CDC). Adverse childhood experiences reported by adults - five states, 2009. MMWR Morb Mortal Wkly Rep. 2010;59(49):1609-1613.

9. Shonkoff JP, Boyce WT, McEwen BS. Neuroscience, molecular biology, and the childhood roots of health disparities: building a new framework for health promotion and disease prevention. JAMA. 2009;301(21): $2252-2259$
10. Keeshin BR, Cronholm PF, Strawn JR. Physiologic changes associated with violence and abuse exposure: an examination of related medical conditions. Trauma Violence Abuse. 2012;13(1):41-56.

11. Anda RF, Croft JB, Felitti VJ, et al. Adverse childhood experiences and smoking during adolescence and adulthood. JAMA. 1999;282(17): 1652-1658.

12. Ford ES, Anda RF, Edwards VJ, et al. Adverse childhood experiences and smoking status in five states. Prev Med. 2011;53(3):188-193.

13. Fuller-Thomson E, Filippelli J, Lue-Crisostomo CA. Gender-specific association between childhood adversities and smoking in adulthood: findings from a population-based study. Public Health. 2013;127(5): 449-460.

14. Anda RF, Brown DW, Dube SR, Bremner JD, Felitti VJ, Giles WH. Adverse childhood experiences and chronic obstructive pulmonary disease in adults. Am J Prev Med. 2008;34(5):396-403.

15. Danese A, Moffitt TE, Harrington H, et al. Adverse childhood experiences and adult risk factors for age-related disease: depression, inflammation, and clustering of metabolic risk markers. Arch Pediatr Adolesc Med. 2009;163(12):1135-1143

16. Dong M, Giles WH, Felitti VJ, et al. Insights into causal pathways for ischemic heart disease: adverse childhood experiences study. Circulation. 2004;110(13):1761-1766.

17. Brown DW, Anda RF, Felitti VJ, et al. Adverse childhood experiences are associated with the risk of lung cancer: a prospective cohort study. BMC Public Health. 2010;10:20.

18. Brown MJ, Thacker LR, Cohen SA. Association between adverse childhood experiences and diagnosis of cancer. PloS One. 2013;8(6): e65524.

19. Sorheim IC, Johannessen A, Gulsvik A, Bakke PS, Silverman EK, DeMeo DL. Gender differences in COPD: are women more susceptible to smoking effects than men? Thorax. 2010;65(6):480-485.

20. Chen Y, Horne SL, Dosman JA. Increased susceptibility to lung dysfunction in female smokers. Am Rev Respir Dis. 1991;143(6): 1224-1230.

21. Langhammer A, Johnsen R, Gulsvik A, Holmen TL, Bjermer L. Sex differences in lung vulnerability to tobacco smoking. Eur Respir J. 2003;21(6):1017-1023.

22. Gan WQ, Man SF, Postma DS, Camp P, Sin DD. Female smokers beyond the perimenopausal period are at increased risk of chronic obstructive pulmonary disease: a systematic review and meta-analysis. Respir Res. 2006;7:52.

23. Centers for Disease Control and Prevention (CDC). Methodologic changes in the Behavioral Risk Factor Surveillance System in 2011 and potential effects on prevalence estimates. MMWR Morb Mortal Wkly Rep. 2012;61(22):410-413.

24. Lu M, Lyden PD, Brott TG, Hamilton S, Broderick JP, Grotta JC. Beyond subgroup analysis: improving the clinical interpretation of treatment effects in stroke research. J Neurosci Methods. 2005;143(2): 209-216.

25. Rothman KJ. Six persistent research misconceptions. J Gen Intern Med. 2014;29(7):1060-1064.

26. Mercy JA, Saul J. Creating a healthier future through early interventions for children. JAMA. 2009;301(21):2262-2264.

27. Edwards VJ, Anda RF, Gu D, Dube SR, Felitti VJ. Adverse childhood experiences and smoking persistence in adults with smoking-related symptoms and illness. Perm J. 2007;11(2):5-13.

28. Walsh EG, Cawthon SW. The mediating role of depressive symptoms in the relationship between adverse childhood experiences and smoking. Addict Behav. 2014;39(10):1471-1476.

29. US Department of Health and Human Services [homepage on the Internet]. The Health Consequences of Smoking - 50 Years of Progress: A Report of the Surgeon General, 2014. Available from: http://www. surgeongeneral.gov/library/reports/50-years-of-progress/. Accessed August 20, 2014.

30. Szklo M, Nieto FJ. Epidemiology: Beyond the Basics. 2nd ed. Sudbury, Mass: Jones and Bartlett Publishers; 2007. 
International Journal of COPD

\section{Publish your work in this journal}

The International Journal of COPD is an international, peer-reviewed journal of therapeutics and pharmacology focusing on concise rapid reporting of clinical studies and reviews in COPD. Special focus is given to the pathophysiological processes underlying the disease, intervention programs, patient focused education, and self management protocols.

This journal is indexed on PubMed Central, MedLine and CAS. The manuscript management system is completely online and includes a very quick and fair peer-review system, which is all easy to use. Visit $\mathrm{http}: / /$ www.dovepress.com/testimonials.php to read real quotes from published authors.

Submit your manuscript here: http://www.dovepress.com/international-journal-of-copd-journal 